\title{
Bibliometría de la sistemática biológica sobre América Latina durante el siglo XX en tres bases de datos mundiales
}

\section{Layla Michán \& Jorge Llorente-Bousquets}

Departamento de Biología Evolutiva, Museo de Zoología. Facultad de Ciencias, UNAM. Av. Universidad 3000 Circuito Exterior S/N, C.P. 04510. Ciudad Universitaria, México, D.F. Edificio “A” Segundo Piso, Teléfono 555622 48 25; laylamichan@ciencias.unam,jlb@hp.fciencias.unam.mx (Autor responsable).

\author{
Recibido 06-IX-2009. C Corregido 22-IX-2009. Aceptado 22-X-2009.
}

\begin{abstract}
Bibliometry of biological systematics in Latin America during the twentieth century in three global databases. We present a review of the biological systematic research in Latin America during the twentieth century, applying a bibliometric analysis to the information contained in international databases with the largest number of biological records: Biosis (since 1969), CAB (since 1910) and Science Citation Index (since 1900), to recognize certain patterns and trends regarding the document production. We obtained 19079 documents and 1387 journals for Biosis, 14326 and 2537 for CAB, 3257 and 1636 for SCI. Of the documents, $54.6 \%$ related to new species, $15.3 \%$ dealt with morphology, $14.9 \%$ keys, $12.5 \%$ descriptions, $10.6 \%$ cases of synonymies, $6 \%$ new genera, $4.9 \%$ new geographical records, $23.6 \%$ geographical distribution, $4.2 \%$ redescriptions, and 3.6\% with new nomenclatural combinations. The regions mentioned were South America with $11.9 \%$, Central America with 4\% and America (all) with 2.56\%. Nineteen Latin American countries appear, whereas outside this region we found the United States of America with $12.6 \%$ of representation and Canada with $3 \%$. Animals (65.6\%) were the most studied taxa, which was 1.7 times higher than what was published for plants (37\%), 11 times higher than fungi (6\%) and nearly 30 times higher than microorganisms $(2.3 \%)$. Out of the 155 journals that produced $66 \%$ of the papers, $76.5 \%$ were better represented in Biosis, $21.4 \%$ in CAB and $2 \%$ in SCI. Twenty-nine journals published 33\% of the articles, the maximum number of records obtained was $69 \%$ for Biosis, CAB 24\% and 6.9\% for SCI, three (10.3\%) are in biology, $11(37.9 \%)$ in botany, 13 (44.8\%) zoology, and two (6.9\%) paleontology; eight of these journals (27.5\%) were published in Latin America and twenty were indexed in the Science Citation Index. In the last two years more journals of the region that publish on taxonomy have been indexed, but their impact factor is still low. However, the impact factor of a number of Latin American journals that published biodiversity increased with time. Countries that are more interested in studying the Latin American biota from the taxonomic point of view are Brazil, the United States, Argentina and Mexico. The most active institutions in this discipline were the Universidade de São Paulo, Universidad Nacional Autónoma de México, and the Universidade Federal do Rio de Janeiro; together they produced 24\% of the documents. Rev. Biol. Trop. 58 (2): 531-545. Epub 2010 June 02.
\end{abstract}

Key words: taxonomy, bibliometry, Biosis, CAB, Science Citation Index, Latin America.

América Latina es una región megadiversa, seis (cerca del $40 \%$ ) de los 17 países megadiversos se encuentran en esta zona: Brasil, Colombia, Ecuador, México, Perú y Venezuela. Individualmente cada uno de estos países tiene más especies de plantas, vertebrados e invertebrados, que la gran mayoría de las naciones del planeta (Mittermeier \& Goettsch
1992, Mittermeier \& Mittermeier 1997). Los países citados son ricos en biotas, ecosistemas, especies, razas geográficas y gran proporción de endemismos (Mittermeier 1988, FloresVillela 1993, Llorente-Bousquets et al. 1996, Rodríguez et al. 2005, Martínez et al. 2006); los cuales son prioritarios para la conservación de hotspots con megadiversidad que está 
amenazada de extinción (Myers et al. 2000). La zona contiene nueve de las 10 ecorregiones más ricas en especies de plantas del mundo (Rodríguez et al. 2005).

Sin embargo, muchas de las especies de América Latina están siendo afectadas negativamente por actividades humanas, en particular por la conversión de ecosistemas: el 20\% de mamíferos, $12 \%$ de aves y $41 \%$ de anfibios endémicos del Neotrópico han sido clasificados como amenazados de extinción (Rodríguez et al. 2005). Siete de las 25 regiones más ricas en endemismos (que también están amenazadas) del planeta se encuentran en esta área (Martínez et al. 2006). Con la actual crisis de la biodiversidad (solo se ha descrito una parte de ésta; a diario se erosionan decenas de miles de ellas y tal vez se extinguen muchas), se corre el riesgo de que gran cantidad de especies no se describan ni conozcan pues posiblemente no quedará registro de ellas. Estamos en el umbral de un enorme empobrecimiento biótico.

De ahí la trascendencia de la investigación sobre taxonomía biológica de la región: la producción de inventarios bióticos, fomentar los estudios sobre la descripción y clasificación de nuevas especies y otros taxones, los estudios florísticos o faunísticos y los análisis filogenéticos, todas ellas tareas de la sistemática y la biogeografía.

Por estas razones es importante analizar y monitorear la investigación taxonómica sobre la biota latinoamericana. Para ello aplicamos análisis bibliométricos a la información documental contenida en tres de las bases de datos mundiales (también llamadas internacionales) con la mayor cantidad de registros sobre taxonomía: Biosis, CAB Abstracts y Science Citation Index (SCI). El propósito es reconocer las características y tendencias respecto a la producción taxonómica sobre Latinoamérica; para lograr este objetivo obtuvimos: 1) Las proporciones de los documentos respecto a las revistas, enfoques, taxones, países estudiados, países e instituciones de adscripción de los autores y la tendencia temporal de la producción en las bases de datos. Identificamos: 2) las revistas más productivas y representativas en cuanto a producción y temas; 3) las revistas y los documentos con mayor impacto (sensu $\mathrm{SCI}$ ); y 4) los países e instituciones más representativos.

\section{MATERIALES Y MÉTODOS}

La investigación consistió en la búsqueda, recuperación, migración, sistematización, homogeneización, normalización, validación, análisis y visualización de la información en términos gráficos (Fig. 1).

En la obtención de información se utilizaron las tres bases de datos internacionales que contienen mayor cantidad de registros sobre sistemática de América Latina: Biosis Previews (Thomson Reuters 2008a) especializada en ciencias biológicas y biomédicas con información desde 1969; CAB Abstracts (CAB) con documentos desde 1910 sobre agricultura y medio ambiente (Commonwealth Agricultural Bureaux (CABI) 2008); Science Citation Index Expanded (SCI) (Thomson Reuters 2008b), que es la base de datos sobre ciencia de corriente principal con registros desde 1900. De cada una de las bases de datos se recuperaron todos los registros sobre taxonomía y sistemática relativos a los países latinoamericanos, la consulta de búsqueda consistió en las palabras: taxonomía y/o sistemática, los nombres de los países de América Latina y "Latin America" para los campos "title", "abstract", "key words", "major concepts" (en Biosis) y "descriptor" (en CAB). La extracción de información se empezó en enero de 2007 y se terminó en abril de 2008, de manera que los resultados están actualizados hasta esa fecha (Cuadro 1). La información se migró y sistematizó en una base de datos relacional; ésta representa en una estructura lógica toda la información extraída, y a su vez facilita la realización de formularios y consultas que permiten un manejo adecuado, un procesamiento eficaz y el análisis consistente. En cada etapa se depuró, se normalizó y se validó la información.

A continuación, se realizaron las consultas y la construcción de matrices de datos que se utilizaron para hacer los análisis estadísticos 


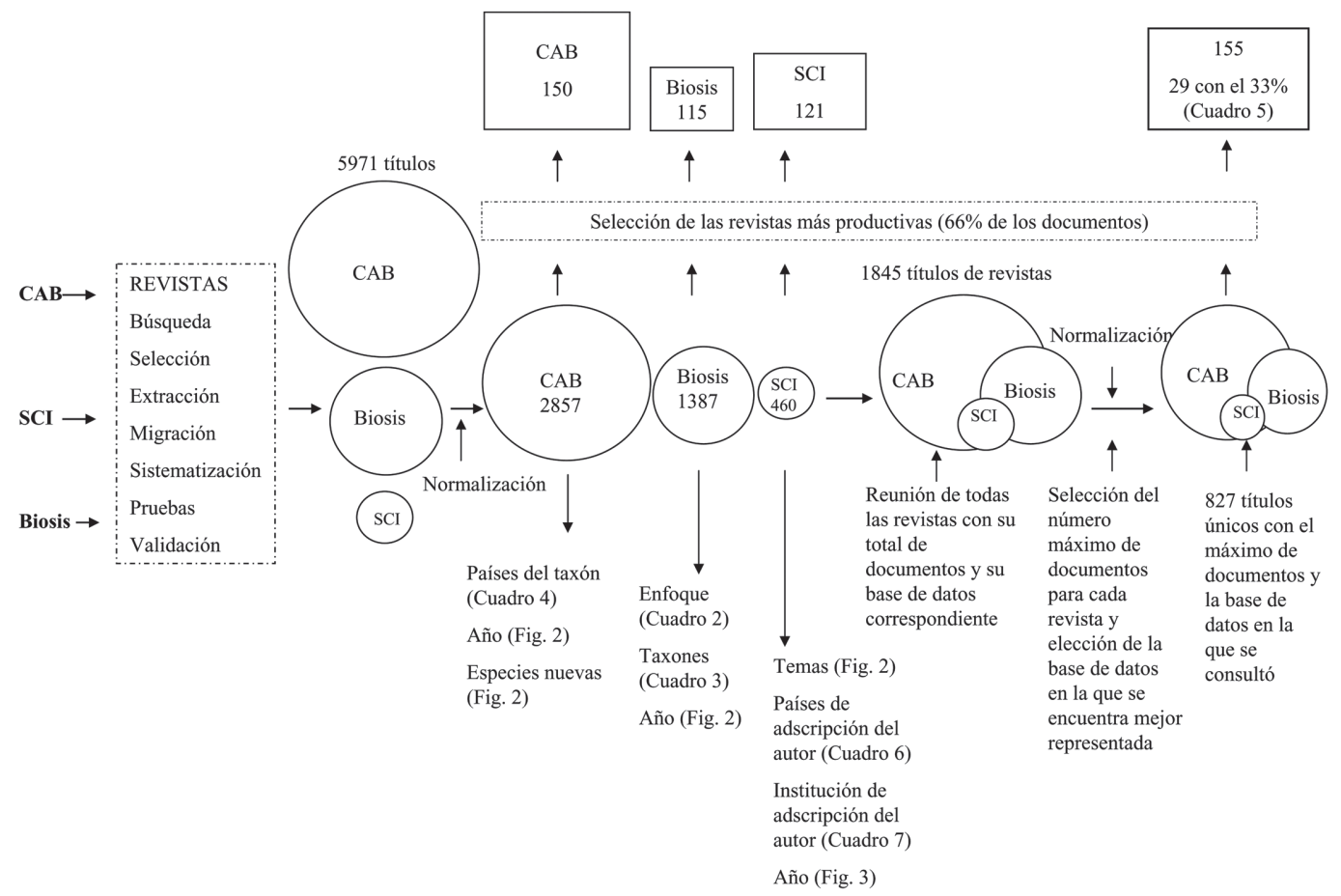

Fig. 1. Diagrama del protocolo de la investigación.

Fig. 1. Diagram of the research protocol.

\section{CUADRO 1}

Registros obtenidos para cada base de datos analizada después de la normalización

TABLE 1

Records for each database analyzed after normalization

$\begin{array}{lccr} & \text { Biosis } & \text { CAB } & \text { SCI } \\ \text { Documentos (principalmente artículos) } & 19079 & 14326 & 3257 \\ \text { Títulos (principalmente revistas) } & 1387 & 2857 & 460 \\ \text { Revistas clave* } & 115 & 150 & 121 \\ \text { Coeficiente de correlación CAB** } & 0.65 & & \\ \text { Coeficiente de correlación con SCI** } & 0.42 & 0.34\end{array}$

*Son las revistas más productivas, corresponden al $66 \%$ de los artículos publicados en cada base de datos analizada.

**El coeficiente de correlación: medida de la intensidad de la relación lineal entre las dos bases de datos referidas en cada caso, puede tomar valores desde menos uno hasta uno, entre más cercano a uno, en cualquier dirección, más fuerte será la asociación lineal entre ellas. 
y los gráficos respectivos en una hoja de cálculo.

Se recuperaron 5971 títulos de revistas de las tres bases de datos, se homogeneizaron y normalizaron los registros quedando 5560 y se eligieron las publicaciones periódicas más productivas para cada una de las tres bases de datos aplicando el criterio de Bradford (Gorbea 1996), que corresponde al 66\% de los documentos (Fig. 1).

Por otro lado, se reunieron todas las revistas (1845), se normalizaron y se reconocieron en total 827 títulos de revistas únicos que se usaron para construir una matriz con la cantidad de artículos y su base de datos correspondiente. Se seleccionó el número máximo de documentos para cada revista y se eligió la base de datos en la que se encuentra mejor representada. Posteriormente, se eligieron las revistas clave totales de acuerdo con Bradford (Gorbea 1996). Se hicieron análisis de los documentos respecto a los campos: año, descriptores y adscripción del autor (Fig. 1).

\section{RESULTADOS}

En el Cuadro 1 se presentan los resultados de la producción contenida en las tres bases de datos analizadas. En cuanto a los títulos corresponden a las publicaciones periódicas: revistas (principalmente), ocasionalmente series, catálogos, monografías y memorias de congresos, por mencionar las más abundantes. Las revistas clave se refieren a aquellas que publicaron las dos terceras partes de los documentos (Bradford 1948). También se presenta el coeficiente de correlación de las revistas de cada base de datos. Los documentos registrados en su mayoría fueron artículos; en SCI éstos constituyeron el $93.5 \%$ del total de los registros, en Biosis $91.5 \%$ y en CAB $88.5 \%$. El resto de los documentos correspondieron a cerca de 20 categorías distintas, entre las más frecuentes estuvieron memorias de congresos, libros y capítulos de libros.

Respecto al idioma de publicación en la base de datos Biosis los documentos más frecuentes fueron $72.4 \%$ en inglés, $13.2 \%$ en español, $9.6 \%$ en portugués y $1.1 \%$ en francés; en $\mathrm{CAB}$ los porcentajes fueron de $58,20,12$, 7 y $1.8 \%$, respectivamente, y de SCI $88.8,6.5$, 3.6 y $0.9 \%$, para cada idioma.

En la Figura 2 se muestra la tendencia temporal (en escala logarítmica) de los registros de las bases de datos analizadas; también se presenta la cantidad de documentos que contienen descripciones de especies nuevas por año en CAB.

En la Figura 3 se grafican los principales temas taxonómicos (cada uno con más del $6 \%$ de representación) de las revistas obtenidas para cada base de datos.

En cuanto al análisis del contenido de los documentos con base en los descriptores, de acuerdo con $\mathrm{CAB}$, el $54.6 \%$ se refieren a especies nuevas, $15.3 \%$ tratan sobre morfología, $14.9 \%$ son claves para 'identificación', $12.5 \%$ descripciones, $10.6 \%$ casos de sinonimias, $6 \%$ géneros nuevos, $4.9 \%$ registros geográficos nuevos, $23.6 \%$ distribución geográfica, $4.2 \%$ redescripciones y $3.6 \%$ son sobre combinaciones nomenclaturales nuevas. En Biosis de acuerdo con sus descriptores ("major concepts"), se reconocen los siguientes porcentajes: $80 \%$ de los documentos abordan nomenclatura y terminología, $39.8 \%$ anatomía e histología, $31.6 \%$ ecología, y $27.3 \%$ versan sobre distribución animal.

Del análisis de los descriptores de los documentos también se obtuvieron los registros de enfoques, taxones y países más frecuentes (Cuadros 2-4). En los Cuadros 2 y 3 se muestran todos aquellos temas y taxones que tuvieron una representación mayor al 1\% en Biosis.

En el Cuadro 4 se presenta los 19 países más frecuentes en los que se localizaron los organismos estudiados en la base de datos CAB. Las regiones de América mencionadas fueron: América del Sur con $11.9 \%$, América Central con $4 \%$ y América (toda) con $2.56 \%$. En cuanto a los países que no pertenecen a esta división política estuvieron Estados Unidos de América con una frecuencia de $12.6 \%$ y Canadá con $3 \%$, esto posiblemente debido a su cercanía, a que comparten intereses comunes y a 


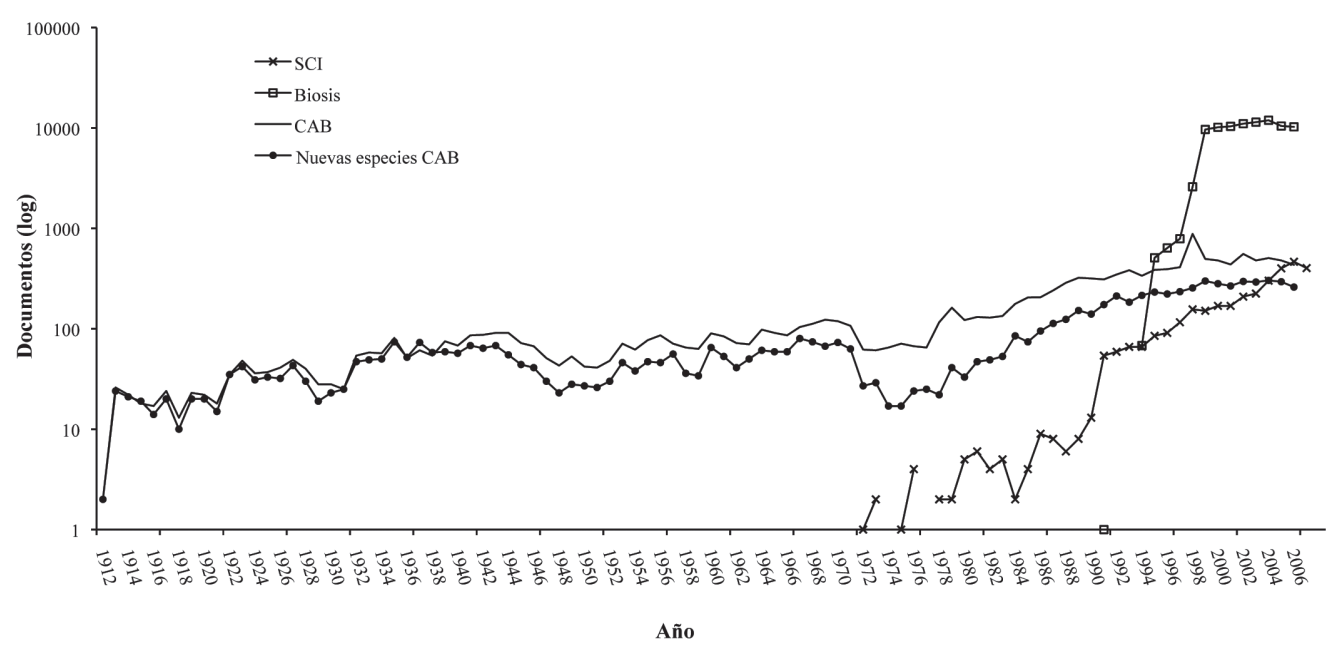

Fig. 2. Tendencia temporal de los documentos de las bases de datos analizadas.

Fig. 2. Temporal trend of the documents of the databases analyzed.

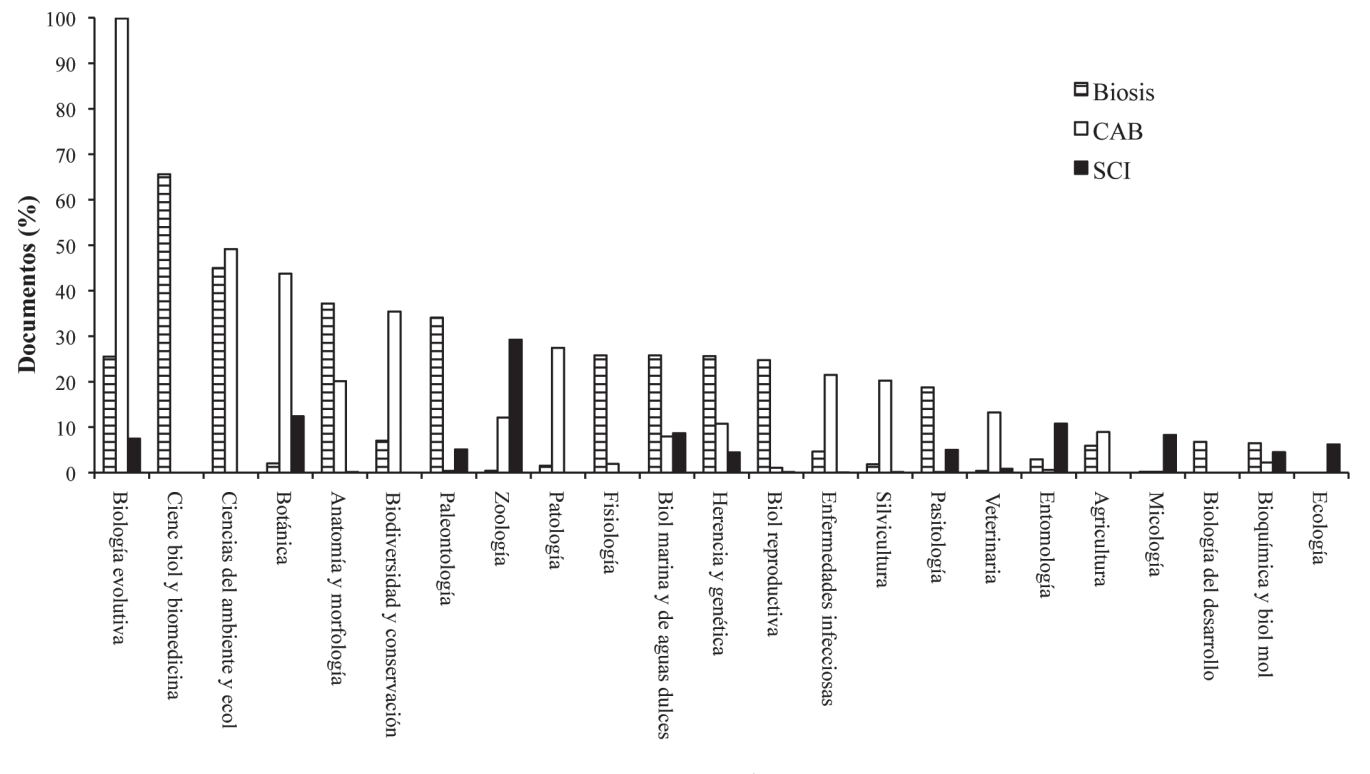

Temas de las revistas

Fig. 3. Principales temas (campo "subject area") para cada base de datos.

Fig. 3. Principal subjects (field "subject area") for each of the databases. 
CUADRO 2

Enfoques más frecuentes en los documentos sobre sistemática biológica de América Latina de acuerdo con el campo "major concepts" de Biosis

TABLE 2

More frequent topics in the papers on systematic biological Latin America under the "major concept" of Biosis

$\begin{array}{lc}\text { Enfoque } & \text { Documentos (\%) } \\ \text { Biogeografía } & 48.50 \\ \text { Morfología } & 18.85 \\ \text { Estudios sobre seres vivos en general } & 12.14 \\ \text { Ecología } & 8.59 \\ \text { Paleobiología } & 7.81 \\ \text { Fisiología } & 5.90 \\ \text { Evolución y adaptación } & 4.88 \\ \text { Ecología terrestre } & 4.79 \\ \text { Parasitología } & 4.66 \\ \text { Sistema reproductivo } & 3.46 \\ \text { Ecología marina } & 3.31 \\ \text { Desarrollo } & 2.94 \\ \text { Genética } & 2.53 \\ \text { Bioquímica y biofísica molecular } & 2.44 \\ \text { Ecología de agua dulce } & 2.25 \\ \text { Genética molecular } & 2.25 \\ \text { Conservación } & 1.94 \\ \text { Manejo de vida silvestre } & 1.90 \\ \text { Comportamiento } & 1.86 \\ \text { Infecciones } & 1.86 \\ \text { Biodiversidad } & 1.71 \\ \text { Genética de poblaciones } & 1.67 \\ \text { Biología celular } & 1.26 \\ \text { Reproducción } & 1.25 \\ \text { Climatología } & 1.20 \\ \text { Nutrición } & 1.14 \\ \text { Ciencias del suelo } & 1.12 \\ & \end{array}$

que muchos estudios taxonómicos comprenden áreas y grupos sin fronteras políticas.

De las 155 revistas que produjeron el $66 \%$ de los artículos, $76.5 \%$ están mejor representadas en Biosis, $21.4 \%$ en CAB y solo $2.0 \%$ en SCI. Nótese la escasa representación porcentual en la última.

En cuanto a las 29 revistas que se integran como las más productivas porque publicaron el 33\% de los artículos (Cuadro 5), el máximo de registros obtenidos fueron $69 \%$ para Biosis,
CUADRO 3

Taxones más estudiados de acuerdo con el campo 'super taxa' de Biosis en los documentos sobre sistemática biológica de América Latina

TABLE 3

More studied taxa from 'super taxa' of Biosis in the papers on systematic biological Latin America

\begin{tabular}{|c|c|}
\hline Taxón & Documentos (\%) \\
\hline Animalia & 65.59 \\
\hline Invertebrata & 44.97 \\
\hline Plantae & 37.18 \\
\hline Arthropoda & 34.07 \\
\hline Espermatofita & 25.80 \\
\hline Chordata & 25.79 \\
\hline Vertebrata & 25.62 \\
\hline Insecta & 25.53 \\
\hline Angiospermae & 24.73 \\
\hline Dicotiledoneas & 18.74 \\
\hline Pisces & 7.01 \\
\hline Mammalia & 6.78 \\
\hline Monocotiledoneas & 6.47 \\
\hline Fungi & 5.92 \\
\hline Crustacea & 4.36 \\
\hline Chelicerata & 4.18 \\
\hline Reptilia & 3.97 \\
\hline Helmintos & 3.83 \\
\hline Mollusca & 3.41 \\
\hline Aves & 3.33 \\
\hline Algae & 2.94 \\
\hline Amphibia & 2.93 \\
\hline Microrganismos & 2.29 \\
\hline Rodentia & 2.25 \\
\hline Aschelminthes & 2.04 \\
\hline Platyhelminthes & 1.89 \\
\hline Bryophyta & 1.85 \\
\hline Protozoa & 1.72 \\
\hline Bacteria & 1.59 \\
\hline Pteridophyta & 1.53 \\
\hline Eubacteria & 1.50 \\
\hline Primates & 1.39 \\
\hline Gymnospermae & 1.14 \\
\hline Annelida & 1.05 \\
\hline
\end{tabular}

$24 \%$ para $\mathrm{CAB}$ y $6.9 \%$ para $\mathrm{SCI}$; tres $(10.3 \%)$ son de biología, 11 (37.9\%) de botánica, 13 (44.8\%) de zoología, y dos $(6.9 \%)$ de paleontología. Ocho de estas revistas $(27.5 \%)$ son editadas en América Latina. Veinte de ellas 
CUADRO 4

Países más estudiados de acuerdo con el campo 'descriptor' de CAB en los documentos sobre sistemática biológica de América Latina

TABLE 4

Countries surveyed according to the 'descriptor' of $C A B$ in the papers on systematic biological Latin America

$\begin{array}{lc}\text { País del estudio } & \text { Documentos (\%) } \\ \text { Brasil } & 30.18 \\ \text { México } & 20.07 \\ \text { Argentina } & 12.22 \\ \text { Venezuela } & 7.55 \\ \text { Perú } & 6.88 \\ \text { Colombia } & 6.69 \\ \text { Costa Rica } & 6.05 \\ \text { Chile } & 5.05 \\ \text { Ecuador } & 4.80 \\ \text { Panamá } & 4.34 \\ \text { Cuba } & 4.29 \\ \text { Bolivia } & 3.27 \\ \text { Guatemala } & 2.71 \\ \text { Puerto Rico } & 2.44 \\ \text { Paraguay } & 1.96 \\ \text { Uruguay } & 1.80 \\ \text { Honduras } & 1.61 \\ \text { Jamaica } & 1.25 \\ \text { Guyana } & 1.04\end{array}$

estuvieron indizadas en el Science Citation Index (Thomson Reuters 2006); sus factores de impacto aparecen en el Cuadro 5 y el promedio del Factor de Impacto de las revistas fue de 0.778. En los Cuadros 6 y 7 se muestran los países y las instituciones que han publicado la mayor proporción de documentos de corriente principal en cuanto a calidad y relevancia (con altos estándares editoriales, estrictos métodos de selección de artículos y gran impacto) de sistemática biológica sobre taxones de América Latina indizados en el SCI (Thomson Reuters 2008d).

\section{DISCUSIÓN Y CONCLUSIONES}

Biosis y $\mathrm{CAB}$ tuvieron representación sobresaliente en documentos sobre taxonomía de la biota latinoamericana (Cuadro 1).
La primera es la base de datos bibliográfica más extensa y sin duda es la mejor fuente de información sobre el tema para las tres últimas décadas (desde 1969). La segunda tiene menos publicaciones periódicas, pero las ha registrado desde hace casi 100 años, por lo tanto aporta una muestra significativa de todo el siglo por su cobertura temporal amplia. SCI, aunque porcentualmente menor, es una de las pocas herramientas para identificar las revistas y los documentos más relevantes desde un punto de vista bibliométrico con base en la citación y contiene literatura sobre el tema que nos ocupa desde 1966; esto seguramente se debe a que privilegia a las revistas de vanguardia con difusión amplia (Fig. 2). CAB y Biosis comparten el $65 \%$ de los títulos de publicaciones periódicas por su afinidad temática, mientras que SCI y Biosis comparten solamente el 42\% (Cuadro 1); seguramente mucha de la información de la primera está compartida con la segunda, pues ambas son administradas por Thomson Reuters. CAB y SCI comparten solamente el 34\% de los títulos.

La producción científica sobre taxonomía de América Latina se puede analizar para los últimos 94 años con base en los documentos examinados de CAB. Este intervalo de tiempo permite reconocer que la publicación sobre el tema tuvo un aumento exponencial, pues pasó de un promedio de dos decenas a principios de siglo a cuatro a mediados de éste, y para finales del mismo aumentó en un orden de magnitud (250 documentos). El crecimiento mostrado es contrastante si se compara con el obtenido para la taxonomía sobre América Latina publicada exclusivamente en las revistas regionales, aunque creciente en ese caso fue lineal y mantenido (Michán et al. 2008). Este resultado muestra claramente la preferencia de los investigadores, durante las últimas décadas, por publicar en revistas extranjeras; lo cual es producto de las políticas científicas regionales implementadas en varios países, en las que se da mayor valor en las evaluaciones cuando publican en las revistas indizadas en el SCI (v.gr. Sistema Nacional de Investigadores en México). Durante la segunda mitad del siglo el acumulado 
CUADRO 5

Las 29 revistas más productivas sobre sistemática biológica de taxones latinoamericanos

TABLE 5

The 29 most productive journals on biological systematics from Latin American taxa

\begin{tabular}{|c|c|c|c|c|c|}
\hline Título & $\begin{array}{l}\text { Cobertura } \\
\text { temática }\end{array}$ & Documentos & $\begin{array}{c}\text { Documentos } \\
\%\end{array}$ & $\begin{array}{l}\text { Base de } \\
\text { datos }\end{array}$ & FI \\
\hline Memorias do Instituto Oswaldo Cruz (AL) & Biología & 856 & 3.56 & $\mathrm{CAB}$ & 1.208 \\
\hline Brittonia & Botánica & 606 & 2.52 & Biosis & 0.211 \\
\hline IMI Descriptions of Fungi and Bacteria & Botánica & 545 & 2.27 & $\mathrm{CAB}$ & \\
\hline Novon & Botánica & 544 & 2.27 & Biosis & 0.150 \\
\hline Revista Brasileira de Zoologia (AL) & Zoología & 427 & 1.78 & Biosis & $*$ \\
\hline Zootaxa & Zoología & 397 & 1.65 & SCI & 0.612 \\
\hline Journal of Parasitology & Zoología & 335 & 1.39 & $\mathrm{CAB}$ & 1.300 \\
\hline Ameghiniana & Paleontología & 310 & 1.29 & Biosis & 0.573 \\
\hline Mycotaxon & Botánica & 303 & 1.26 & Biosis & 0.486 \\
\hline Revista Brasileira de Entomologia (AL) & Zoología & 282 & 1.17 & Biosis & * \\
\hline Proceedings of the Biological Society of Washington & Biología & 277 & 1.15 & Biosis & 0.347 \\
\hline Revista de Biología Tropical (AL) & Biología & 271 & 1.13 & Biosis & 0.217 \\
\hline $\begin{array}{l}\text { Proceedings of the Entomological Society of } \\
\text { Washington }\end{array}$ & Zoología & 267 & 1.11 & Biosis & 0.394 \\
\hline Journal of Vertebrate Paleontology & Paleontología & 220 & 0.92 & Biosis & 1.418 \\
\hline Iheringia Serie Zoologia (AL) & Zoología & 204 & 0.85 & Biosis & $*$ \\
\hline Herpetologica & Zoología & 183 & 0.76 & $\mathrm{SCI}$ & 1.019 \\
\hline Revista Brasileira de Biologia (AL) & Biología & 174 & 0.72 & $\mathrm{CAB}$ & \\
\hline Coleopterists Bulletin & Zoología & 168 & 0.70 & Biosis & 0.397 \\
\hline Copeia & Zoología & 166 & 0.69 & Biosis & 0.840 \\
\hline Systematic Parasitology & Zoología & 163 & 0.68 & $\mathrm{CAB}$ & 0.856 \\
\hline Annals of the Entomological Society of America & Zoología & 150 & 0.62 & $\mathrm{CAB}$ & \\
\hline SIDA Contributions to Botany & Botánica & 144 & 0.60 & Biosis & \\
\hline Kew Bulletin & Botánica & 143 & 0.60 & Biosis & \\
\hline Folia Entomológica Mexicana (AL) & Zoología & 142 & 0.59 & Biosis & \\
\hline Systematic Botany & Botánica & 137 & 0.57 & Biosis & 1.629 \\
\hline Hoehnea & Botánica & 136 & 0.57 & Biosis & \\
\hline Mycologia & Botánica & 129 & 0.54 & $\mathrm{CAB}$ & 1.579 \\
\hline Studies on Neotropical Fauna and Environment & Zoología & 123 & 0.51 & Biosis & \\
\hline Memoirs of the New York Botanical Garden & Botánica & 122 & 0.51 & Biosis & \\
\hline
\end{tabular}

FI: Factor de Impacto calculado por el ISI para el 2006 (Thomson Reuters 2008c).

(AL). Las revistas editadas en países de América Latina.

1. Se refiere a la base de datos en la que está mejor representada esta revista.

* Actualmente están siendo indizadas en el SCI pero hasta después de dos años se calcula el índice de impacto.

de documentos fue de 4.5 veces más que la primera mitad, pues aumentó de 2468 a 11 316. El año de 1998 resaltó por su producción con 880 registros; este aumento posiblemente coincida con la organización de congresos de sociedades internacionales y la publicación de sus memorias (Fig. 2). El resultado es representativo de la producción sobre taxonomía de la región en revistas internacionales, en especial si se considera que para este trabajo se han utilizado las bases de datos internacionales que 
CUADRO 6

Países de adscripción de los autores que publican la mayor cantidad de documentos de corriente principal en sistemática biológica sobre América Latina según SCI

TABLE 6

Countries of assignment of the authors who publish the largest number of papers in mainstream systematic biology from Latin America as SCI

$\begin{array}{lc}\text { País del autor } & \text { Documentos (\%) } \\ \text { Brasil } & 44.89 \\ \text { Argentina } & 20.33 \\ \text { EUA } & 19.16 \\ \text { México } & 17.41 \\ \text { Chile } & 5.68 \\ \text { España } & 4.67 \\ \text { Inglaterra } & 4.36 \\ \text { Venezuela } & 3.29 \\ \text { Alemania } & 3.16 \\ \text { Francia } & 3.07 \\ \text { Colombia } & 2.95 \\ \text { Cuba } & 2.76 \\ \text { Costa Rica } & 2.49 \\ \text { Panamá } & 2.21 \\ \text { Canadá } & 1.93 \\ \text { Perú } & 1.93 \\ \text { Bélgica } & 1.66 \\ \text { Holanda } & 1.35 \\ \text { Australia } & 1.14 \\ \text { Suiza } & 1.11 \\ \text { Uruguay } & 1.04\end{array}$

tienen mayor cantidad de documentos sobre el tópico (Michán datos no publicados).

Los temas que privilegia cada base de datos se pueden reconocer en la Figura 3, este resultado es útil para estudiar las tendencias de producción en cada área y establecer cuáles catálogos utilizar en la búsqueda respecto a alguna disciplina, enfoque, taxón u objeto de estudio específico. Por ejemplo CAB es idónea para botánica y Biosis para paleontología.

Más detalles sobre la producción científica se obtuvieron a partir del análisis del contenido de la literatura recuperada, para ello se utilizaron las palabras clave que registran Biosis ('Major concept' y 'Major Taxa') (Thomson Reuters 2008a) y CAB ('Descriptors') para cada documento (CABI 2008). En la primera se refieren a las áreas temáticas incluidas en el documento original por el autor y a las jerarquías taxonómicas (en orden decreciente) de cada registro; el examen del primer campo permitió reconocer los enfoques principales y el segundo los taxones. CAB recurre a los descriptores que son asignados por especialistas con base en el contenido de cada escrito, para ello utilizan como referencia un tesauro (lista de términos controlados llamados 'Descriptors') que se usa en la clasificación de los documentos; para nuestros propósitos fueron adecuados en la identificación de los países o regiones en los que se localizan los taxones objeto de los estudios sistemáticos, los temas más recurrentes y las descripciones de taxones nuevos.

Estos campos resultaron de gran utilidad para la investigación bibliométrica, pues permitieron hacer análisis más detallados respecto a los temas, taxones y regiones que tratan los documentos. Son pocos los estudios infométricos que utilizan estas bases de datos y, menos aún, aquellos sobre algún tema afín que hayan utilizado estos campos para realizar el análisis de las revistas. Esta información constituye una buena estrategia para recuperar documentos eficientemente, ya que permite identificarlos de una manera más precisa.

Enfoques: De acuerdo con Biosis los enfoques o temas taxonómicos más repetidos en la investigación taxonómica sobre la biota latinoamericana fueron la biogeografía sensu lato (distribución en general), la morfología, la ecología y la paleobiología (Cuadro 2). La bioquímica, la biofísica y la genética solo aparecen representadas en cerca del $2.5 \%$ de los documentos respectivamente; este porcentaje es alto comparado con los resultados obtenidos del análisis de lo publicado en revistas de América Latina exclusivamente, pues las dos primeras disciplinas están por debajo del $0.5 \%$ y la última aparece con $1.3 \%$ (Michán et al. 2008). Este resultado indica que tales estudios son publicados con mayor frecuencia en revistas de mayor difusión e impacto. El 
CUADRO 7

Instituciones de adscripción de los autores que publicaron mayor cantidad de documentos de corriente principal de sistemática biológica sobre América Latina según SCI

TABLE 7

Institutions of assignment of the authors with largest number of papers in mainstream systematic biology from Latin America as SCI

$\begin{array}{lc}\text { Institución del autor } & \text { Documentos (\%) } \\ \text { Universidade de São Paulo (Brasil) } & 9.70 \\ \text { Universidad Nacional Autónoma de México (México) } & 7.92 \\ \text { Universidade Federal do Rio de Janeiro (Brasil) } & 6.69 \\ \text { Universidad de Buenos Aires (Argentina) } & 3.41 \\ \text { Consejo Nacional de Investigaciones Científicas y Técnicas (Argentina) } & 3.29 \\ \text { Universidade Federal do Paraná (Brasil) } & 3.07 \\ \text { Museo de La Plata (Argentina) } & 2.12 \\ \text { Smithsonian Tropical Research Institute (EUA) } & 2.03 \\ \text { Instituto Oswaldo Cruz (Brasil) } & 1.96 \\ \text { Universidade Federal do Rio Grande do Sul (Brasil) } & 1.93 \\ \text { Instituto de Ecología, A. C. (México) } & 1.81 \\ \text { Fundação Oswaldo Cruz (Brasil) } & 1.72 \\ \text { Universidade Federal de Minas Gerais (Brasil) } & 1.63 \\ \text { Universidad Federal de Vicosa (Brasil) } & 1.50 \\ \text { Universidad Nacional de Córdoba (Argentina) } & 1.47 \\ \text { Universidad Nacional de La Plata (Argentina) } & 1.44 \\ \text { Universidade Estadual de Campinas (Brasil) } & 1.41 \\ \text { Universidade Estadual Paulista (Brasil) } & 1.38 \\ \text { Instituto Butantan (Brasil) } & 1.29 \\ \text { Universidad de Costa Rica (Costa Rica) } & 1.26 \\ \text { United States Department of Agriculture (EUA) } & 1.23 \\ \text { Universidad Austral de Chile (Chile) } & 1.20 \\ \text { Consejo Superior de Investigaciones Científicas (España) } & 1.14 \\ \text { Instituto Politécnico Nacional (México) } & 1.07 \\ \text { Universidade Federal do Paraná (Brasil) } & 1.07 \\ \text { Natural History Museum (Inglaterra) } & 1.07 \\ & \end{array}$

análisis de los temas en $\mathrm{CAB}$ permitió identificar que correspondieron a nuevas especies $29.1 \%$ de los documentos, trataron sobre evolución $16 \%$, morfología $15.5 \%$, distribución geográfica $12.9 \%$, filogenética $10.7 \%$, filogenia $10 \%$, claves de identificación de especies $9.8 \%$, descripciones en general $9.6 \%$, nuevos registros geográficos $5.1 \%$ y nomenclatura $4.8 \%$, todas ellas tareas primordiales de la taxonomía y sus consecuencias distribucionales o biogeográficas.

La proporción de documentos detectada que describe especies nuevas (29\%) fue ligeramente mayor al obtenido para lo publicado en América Latina (24.8\%) sobre la biota de la misma área (Michán et al. 2008); no obstante es casi igual a lo publicado en México (30\%) sobre taxones mexicanos en TaXMeXX (Michán y Llorente 2003). De tal manera que, al parecer, cerca de una tercera parte de los documentos sobre sistemática biológica de países de Latinoamérica publican al menos la descripción de una especie nueva.

La tendencia temporal de publicación de artículos con descripción de especies nuevas fue similar a la producción taxonómica en $\mathrm{CAB}$ 
(Fig. 2), aunque la proporción de artículos con descripciones disminuyó con el paso del tiempo. Este resultado puede ser natural pues cada vez es más difícil descubrir especies nuevas, en especial de grupos con tradición y tomando en cuenta que el número de taxónomos, aunque ha crecido (no al ritmo deseado), se dedica también a otras tareas de la sistemática biológica.

Taxones: Los taxones más estudiados durante las últimas tres décadas, de acuerdo con Biosis, fueron por mucho los animales (65.6\%) que representaron 1.7 veces más de lo que se publicó para plantas (37\%), 11 veces más que los hongos $(6 \%)$ y cerca de 30 veces más que los microorganismos $(2.3 \%)$ (Cuadro 3 ). Los grupos mejor representados fueron claramente los invertebrados, en especial los artrópodos. Todos estos resultados eran de esperarse pues son aproximadamente consistentes con la riqueza de especies de cada taxón superior, excepto el caso de las plantas, que tienen un porcentaje muy por encima de su riqueza de especies proporcional respecto de los insectos (subgrupo de artrópodos). Estos últimos constituyen el $65 \%$ de la diversidad alfa de cualquier biotipo terrestre; las plantas solo conforman el $15 \%$. Esto puede indicar que hay otras razones más allá de la simple riqueza de especies para favorecer el estudio de un taxón, como su aplicación médica o industrial, o bien el mayor número proporcional de taxónomos, como es el caso de México.

Países y regiones de estudio: Respecto a los países y las regiones de América Latina a los que se refirieron los taxones estudiados, de acuerdo con $\mathrm{CAB}$, se mencionaron 19 países latinoamericanos, la mayoría (62\%) se concentraron en tres de ellos: Brasil (30\%), México (20\%) y Argentina (12\%) (Cuadro 4).

Las revistas: Dentro de las 29 revistas más productivas en sistemática biológica (Cuadro 5) ocho fueron editadas en la región; las Memorias do Instituto Oswaldo Cruz, brasileña, fundada en 1909, es la publicación con mayor número de documentos y la que tiene el mayor impacto de las revistas regionales, tal resultado la convierte, sin duda, en la revista de mayor tradición de taxonomía de la región. Para botánica resaltaron tres revistas extranjeras: Brittonia, IMI Descriptions of Fungi and Bacteria y Novon, y para zoología una regional y una internacional especializada en sistemática: Revista Brasileira de Zoología y Zootaxa. En el Cuadro 5 se puede identificar en cuál base de datos está mejor representada cada una de estas revistas, dato que puede resultar útil para elegir el catálogo idóneo para recuperar información sobre alguna de ellas. Este mismo cuadro podría ser útil para identificar las revistas y las bases de datos adecuadas para hacer análisis más detallados de cada uno de los temas. Cabe aclarar que estos resultados son válidos únicamente para el período aquí estudiado.

El Factor de Impacto (FI) de las revistas es publicado anualmente por Thomson Reuters en los Journal Citation Reports, uno de los indicadores bibliométricos más consultado por su aplicación en evaluación de la ciencia. Éste se obtiene del cociente del total de citas obtenidas por cada revista durante el año al que se refiere (por ejemplo 2006) en documentos publicados los dos años previos (2004-2005), entre el total de documentos publicados por esa misma revista durante tal periodo bianual. Dentro de las revistas que publicaron documentos sobre sistemática de la biota latinoamericana, una especializada en sistemática, Systematic Botany tuvo el FI mayor con 1.629 (Cuadro 5). En el SCI no existe la categoría disciplinar sistemática o taxonomía, pero hay información respecto al FI de varias disciplinas relacionadas con la sistemática; para el 2006 (Thomson Scientific 2008) fueron en orden decreciente: biología evolutiva 2.491 , micología 1.574, biología marina y de aguas dulces 1.196 , biología 1.138 , ciencias ambientales 1.129 , botánica 0.971 , paleontología 0.942 , zoología 0.924 , conservación de la biodiversidad 0.887 , entomología 0.727 y ornitología 0.660 ; el promedio de todas ellas es 1.149. Al utilizar como referencia estas cifras se puede decir que el mayor FI lo presenta la biología evolutiva, pues la 
botánica y la zoología presentan índices similares. El FI obtenido para las revistas editadas en América Latina en general fue relativamente bajo: con excepción de las Memorias do Instituto Oswaldo Cruz, todas tuvieron un número menor a 0.6 (Cuadro 5). En los últimos dos años se han indizado más revistas editadas en la región que publican sobre taxonomía, pero su FI aún sigue siendo bajo. Sin embargo, varias de las revistas latinoamericanas que publicaron sobre biodiversidad aumentaron este indicador con los años, por ejemplo, esta misma revista tuvo un FI para los años anteriores de: 1.208 (2006), 0.847 (2005), 0.740 (2004), 0.688 (2003) y 0.635 (2002), posiblemente como resultado de la consolidación de la institucionalización de la disciplina en la región, o el aumento del número de taxónomos (campana de resonancia).

Las instituciones y los países: El examen de las instituciones y los países de adscripción de los autores de los artículos de corriente principal (internacionales) de sistemática sobre América Latina, indizados en SCI, nos proporcionaron información respecto a cuáles fueron, desde los años 60s, los países y las instituciones que realizaron más investigación sobre taxonomía de esta región. Los países que más se han interesado en estudiar la biota de América Latina desde el punto de vista taxonómico son: Brasil, con la autoría de casi la mitad de los documentos, lo que representa más del doble que lo registrado para los tres siguientes países: Estados Unidos, Argentina y México (Cuadro 6). Estos resultados son semejantes a la producción obtenida para América Latina sobre la ciencia en general (Cetto \& Vessuri 1998, 2005, De Moya-Anegón \& HerreroSolana 1999, RICYT 2008), la bioquímica y la fisiología (Hermes-Lima \& Navas 2006), las plantas medicinales (Calixto 2005), las ciencias agrícolas (Saavedra-Fernández et al. 2002), y ciencia y tecnología en alimentos. Respecto a la institución más activa en este campo disciplinar se reconoce a la Universidade de São Paulo (brasileña), seguida de la Universidad Nacional Autónoma de México y la Universidade
Federal do Rio de Janeiro (también brasileña); las tres instituciones produjeron el $24 \%$ de los documentos (Cuadro 7).

De acuerdo con los indicadores bibliométricos obtenidos en esta investigación es preponderante el papel de Brasil en el estudio de la taxonomía sobre la biota de América Latina, seguido de México y Argentina. Estos tres países también fueron los más representados en los estudios taxonómicos publicados regionalmente (Michan et al., 2008) y son los que destacan en la mayoría de las áreas del conocimiento científico y humanístico, generalmente en ese mismo orden (UNESCO 2005, OECD 2007, OST 2007, RICYT 2008). Considérese que estos tres países también conforman la mayor parte del PIB de América Latina y del gasto en ciencia y tecnología dentro de la región (De Moya-Anegón \& Herrero-Solana 1999, Hermes-Lima et al. 2007, Zenteno-Savin et al. 2007).

Con base en los resultados se puede concluir que la producción taxonómica sobre un país determinado no depende únicamente de su megadiversidad, como es el caso de las dos primeras naciones, sino también de otros factores, como su tamaño y población, el desarrollo e inversión en ciencia y tecnología; tal es la situación de Brasil que tiene una tradición científica consolidada y que ha hecho esfuerzos interesantes por desarrollar estudios en ciencia y tecnología. Su representación en este ámbito en la ciencia internacional es la de mayor impacto de los países de América Latina. Sería de gran importancia e interés el hacer estudios bibliométricos que incluyan indicadores económicos, de ciencia, tecnología, desarrollo, sociales, poblacionales y educativos, por ejemplo, para tener una visión integral que considere el enfoque interno y externo, conveniente en cualquier estudio de historia de la ciencia (Kragh 1989).

Algunas reflexiones y comentarios: $\mathrm{El}$ análisis bibliométrico sobre la producción científica de alguna región o país puede hacerse desde distintas perspectivas de acuerdo con los elementos involucrados, por ejemplo: 1) el 
contenido, al utilizar como objeto de estudio todos aquellos documentos que traten sobre dicha región, en este caso la biota de distintas áreas de América Latina; 2) los autores, al seleccionar la literatura escrita por investigadores que están adscritos a alguna institución del área a estudiar; 3) las publicaciones, al identificar lo editado en la propia región (revistas endógenas), esto es, todas aquellas publicaciones periódicas editadas por cualquier instancia latinoamericana de lo publicado en el extranjero (revistas exógenas). Este último punto es determinante si se considera que la documentación está sistematizada principalmente en bases de datos que utilizan como unidad de registro las revistas y los artículos que contienen (generalmente gran diversidad de tipos de artículos de investigación). La selección de revistas que indiza cada base de datos se hace aplicando criterios diferentes o distintos de acuerdo con su propósito. Por ejemplo Science Citation Index pone énfasis en que se cumplan de forma muy estricta las normas editoriales, el idioma inglés y el impacto (JCR), pues su fin es registrar las revistas científicas más importantes a nivel mundial. Biosis y CAB pretenden ser exhaustivas en su especialidad de biología básica y aplicada, respectivamente. Por estas razones, todas las bases de datos bibliográficas tienen cobertura distinta: geográfica (el país de edición de la revista), idiomática, temática (disciplinas que incluye) y temporal (periodo de tiempo que abarca la colección) y capturan diferentes tipos de información para cada documento, por mencionar las más importantes. Por lo tanto, cada catálogo contiene información distinta y diversas variables para hacer estudios bibliométricos, esa fue precisamente la ventaja de utilizar bases de datos distintas para nuestro estudio.

La cobertura geográfica e idiomática siempre son elementos importantes a considerar en la investigación bibliométrica, particularmente sobre alguna región distinta a la anglosajona o de primer mundo. Puesto que la representación de las revistas científicas de América Latina en bases de datos mundiales es mínima (Cetto \& Kai-Inge 1995, Plaza 1998, Michán et al.
2008), esto implica que los criterios que se aplican son distintos en la elección de la fuente de información (las bases de datos bibliográficas) y de los documentos a estudiar (las consultas), así como en la sistematización y clasificación de la información (catálogos y relaciones lógicas). Esto representa problemas o dificultades para 'sumar' la información proveniente de los diferentes catálogos y referente a los tres aspectos (biota, investigadores y revistas) en una sola base de datos que permita realizar análisis más completos o integrales.

Hasta donde sabemos, éste es el primer trabajo que explora la producción científica de la taxonomía sobre las especies de América Latina durante el siglo XX e inicios del XXI, utilizando como criterio de selección los documentos de la región sobre los organismos estudiados, y utilizando como fuente las bases de datos mundiales de mayor cobertura de revistas exógenas. Previamente se realizó un estudio empleando como referencia las publicaciones periódicas publicadas en la región, utilizando la base de datos Periódica para estudiar las características generales de los artículos (Michán et al. 2008); en prensa está un estudio sobre las revistas (Michán 2009) y próximamente aparecerá un trabajo sobre los autores que han investigado la biota de la región.

Nuestro siguiente objetivo consiste en integrar la información producida, tanto endógena cómo exógenamente, a partir de bases de datos bibliográficas internacionales (como las aquí presentadas), regionales como Periódica, Scielo y Redalyc y algunas locales o nacionales.

Información sistematizada, completa, consistente y normalizada nos permitiría tener un panorama integral del desarrollo de los estudios sobre la diversidad de organismos de la región, a partir de los cuales podríamos responder a preguntas tales como: ¿qué países y regiones han sido los que más han contribuido al conocimiento de la región?, ¿en la actualidad estudian más su biodiversidad los científicos locales?, ¿qué instituciones han sido la más relevantes?, ¿qué revistas contienen la mayor cantidad de información y sobre qué regiones y taxones tratan?, ¿cuál es la dinámica de la colaboración 
en el área?, ¿cuáles son los temas de interés de cada región y disciplina?, ¿cuál es el ritmo de descripción de nuevas especies y taxones?, por mencionar algunas de las cuestiones más importantes.

También sería de interés integrar al análisis bibliométrico los libros, las monografías y los capítulos publicados sobre la biota de América Latina, dado que éstos constituyen documentos de referencia básica para la investigación sobre diversidad de especies, pero muy pocos están registrados en las bases de datos bibliográficas, y no existe, hasta el momento, ningún catálogo que los sistematice para hacer tales compilaciones y análisis bibliométricos. Por ende, sería conveniente diseñar una base de datos de capítulos, monografías y libros sobre estudios de sistemática biológica de América Latina que incluya la información bibliográfica completa, incluidas las referencias; de modo que se cuente con una herramienta de consulta que también sirva para localizar la bibliografía que aún se encuentra dispersa.

\section{AGRADECIMIENTOS}

A Armando Luis e Isabel Vargas por el apoyo que nos brindaron para la realización de la investigación. A Minerva García Palacios por su ayuda computacional. Jimena Castro nos colaboró con el diseño de la Figura 1. Dos revisores anónimos nos dieron sugerencias sobre el manuscrito. Este trabajo se realizó gracias al soporte de los programas PROFIP-DGAPA a Layla Michán, los proyectos PAPIIT IN203509 y CONACYT 83237 a Jorge Llorente.

\section{RESUMEN}

Aplicamos bibliometría a las bases de datos: Biosis, $\mathrm{CAB}$ y Science Citation Index. El $54.6 \%$ de los documentos son especies nuevas, $15.3 \%$ morfología, $14.9 \%$ claves, $12.5 \%$ descripciones, $10.6 \%$ sinonimias, $6 \%$ géneros nuevos, $4.9 \%$ registros geográficos nuevos, $23.6 \%$ distribución geográfica, $4.2 \%$ redescripciones y 3.6 combinaciones nomenclaturales nuevas. Los taxones más estudiados fueron los animales $(65.6 \%)$, seguidos de las plantas (37\%), los hongos (6\%) y los microorganismos $(2.3 \%)$. Veintinueve revistas publicaron el $33 \%$ de los artículos, tres (10.3\%) son de biología, $11(37.9 \%)$ de botánica, 13 (44.8\%) de zoología y dos (6.9\%) de paleontología; ocho revistas $(27.5 \%)$ son editadas en la región y veinte estuvieron indizadas en el SCI. En los últimos dos años se han indizado más revistas editadas en la región que publican sobre taxonomía, pero su factor de impacto sigue siendo bajo respecto al promedio del área. Los países que más se han interesado en estudiar la biota de América Latina desde el punto de vista taxonómico son: Brasil, Estados Unidos, Argentina y México. Las instituciones más activas fueron la Universidade de São Paulo, la Universidad Nacional Autónoma de México y la Universidade Federal do Rio de Janeiro; juntas produjeron el $24 \%$ de los documentos.

Palabras clave: taxonomía, ciencimetría, Biosis, CAB, Science Citation Index, América Latina.

\section{REFERENCIAS}

Bradford, S.C. 1948. Documentation. Crosby Lockwood, Londres, Inglaterra.

Calixto, J.B. 2005. Twenty-Five Years of Research on Medicinal Plants in Latin America - A Personal View. J. Ethnopharmacol. 100: 131-134.

Cetto, A.M. \& H. Kai-Inge. 1995. Publicaciones científicas en América Latina. FCE, ICSU, UNESCO, UNAM y AIC, México, Distrito Federal, México.

Cetto, A.M. \& H. Vessuri. 1998. Latin America and the Caribbean, 55-75 p. In World Science Report 1998. UNESCO, Paris, Francia.

Cetto, A.M. \& H. Vessuri. 2005. Latin America and the Spanish-speaking Caribbean, p. 45-76. In World Science Report 2005. UNESCO, París, Francia.

De Moya-Anegón, F. \& V. Herrero-Solana. 1999. Science in America Latina: A comparison of bibliometric and scientific-technical indicators. Scientometrics 46: 299-320.

Flores-Villela, O. 1993. Herpetofauna of Mexico: Distribution and Endemism, p. 253-280. In T. P. Ramamoorthy, R. Bye, A. Lot \& J. Fa (eds.). Biological Diversity of Mexico: Origins and Distributions. Oxford University Press, Nueva York, Nueva York, EEUU.

Gorbea, S. 1996. El Modelo Matemático de Bradford: $\mathrm{Su}$ aplicación a las revistas latinoamericanas de las ciencias bibliotecológicas y de la información. Centro Universitario de Investigaciones Bibliotecológicas, UNAM, México, Distrito Federal, México.

Hermes-Lima, M., A.C.R. Alencastro, N.C.F. Santos, C.A. Navas \& R.O. Beleboni. 2007. The Relevance and Recognition of Latin American Science. Introduction 
to the fourth issue of CBP-Latin America. Comp. Biochem. Physiol. Toxicol. \& Pharmacol. 146: 1-9.

Hermes-Lima, M. \& C.A. Navas. 2006. The Face of Latin American Comparative Biochemistry and Physiology. Comp. Biochem. Physiol. Toxicol. \& Pharmacol. 142: $157-162$.

Kragh, H. 1989. Introducción a la historia de la ciencia. Crítica, Barcelona, España.

Llorente-Bousquets, J., E. González, A.N. García-Aldrete \& C. Cordero. 1996. Breve Panorama de la Taxonomía de Artrópodos en México, p. 3-14. In J. Llorente Bousquets et al., (eds.). Biodiversidad, Taxonomía y Biogeografía de Artrópodos de México: Hacia una Síntesis de su Conocimiento. México, Instituto de Biología, UNAM, México, Distrito Federal, México.

Martínez, M.L., R.H. Manson, P. Balvanera, R. Dirzo, J. Soberón, L. García-Barrios, M. Martínez-Ramos, P. Moreno-Casasola, L. Rosenzweig \& J. Sarukhán. 2006. The Evolution of Ecology in Mexico: Facing Challenges and Preparing for the Future. Front. Ecol. Environ. 5: 259-267.

Michán, L. 2009. Las revistas y la institucionalización de la sistemática en Latinoamérica. Rev. Mex. Biodiv. 80: 105-117.

Michán, L. \& J. Llorente. 2003. La taxonomía en México durante el siglo XX. Publ. Esp. Mus. Zool. 13: $1-250$.

Michán, L., J. Russell, A. Sánchez Pereyra, A. Llorens Cruset \& C. López Beltrán. 2008. Análisis de la sistemática actual en Latinoamérica. Interciencia 33: 754-761.

Mittermeier, R.A. 1988. Primate diversity and the tropical forest: case studies from Brazil and Madagascar and the importance of the megadiversity countries, p. 145-154. In E.O. Wilson. Biodiversity. National Academy, Washington, D.C., USA.

Mittermeier, R. \& C. Goettsch. 1992. La importancia de la diversidad biológica de México. Comisión Nacional para el Conocimiento y Uso de la Biodiversidad, México, Distrito Federal, México.

Mittermeier, R.A. \& C. G. Mittermeier.1997. Megadiversity: Earth's Biologically Wealthiest Nations. CEMEX, México, Distrito Federal, México.

Myers, N., R.A. Mittermeier, C.G. Mittermeier, G.A.B. da Fonseca \& J. Kent. 2000. Biodiversity hotspots for conservation priorities. Nature 403: 853-858.

Plaza, L.M. 1998. The use of multiple databases in the assessment of research. An application in the field of plant science. Scientometrics 43: 299-304.
Rodríguez, J.P., T. Good \& R. Dirzo. 2005. Diversitas and the challenge of Latin American biodiversity conservation. Interciencia 30: 450-450.

Saavedra-Fernández, Ó., G. Sotolongo-Aguilar \& M.V. Guzmán-Sánchez. 2002. Medición de la producción científica en América Latina y el Caribe en el campo agrícola y afines: un estudio bibliométrico. Rev. Esp. Doc. Cient. 25: 151-161.

UNESCO. 2005. Science Report 2005. UNESCO, París, Francia.

Zenteno-Savin, T., R.O. Beleboni \& M. Hermes-Lima. 2007. The Cost of Latin American Science - Introduction for the Second Issue of CBP-Latin America. Comp. Biochem. Phys. Mol. \& Integrative Physiol. 146: 463-469.

\section{REFERENCIAS DE INTERNET}

Commonwealth Agricultural Bureaux (CABI). 2008. CAB Abstracts. (Consultado: 15 abril 2008, http://www. cabi.org/datapage.asp?iDocID=165.).

OECD. 2007. Stats: Science, Technology and Patents. (Consultado: 15 abril 2008, http://stats.oecd.org/ wbos/Default.aspx?usercontext=sourceoecd.).

OST. 2007. Chiffres clés de la science et de la technologie, édition 2006. (Consultado: 5 octubre 2007, http:// www.obsost.fr/fileadmin/medias/tx_ostdocuments/ Partie5.pdf).

RICYT. 2008. Red de Indicadores de Ciencia y TecnologíaIberoamericana e Interamericana: Indicadores comparativos. (Consultado: 5 septiembre 2008, http:// www.ricyt.edu.ar/interior/interior.asp?Nivel1 $=1 \& \mathrm{Ni}$ vel2=2\&Idioma $=$.).

Thomson Reuters. 2006. Journal Scitation Report. (Consultado: 8 noviembre del 2008, http://scientific.thomsonreuters.com/products/jcr/.).

Thomson Reuters. 2008a. Biosis Previews. (Consultado: 21 abril 2008, http://www.biosis.org/.).

Thomson Reuters. 2008b. Dialog Bluesheets. (Consultado: 15 mayo 2008, http://library.dialog.com/bluesheets/ html/bli.html.).

Thomson Reuters. 2008c. Journal Scitation Report 2006. (Consultado: 8 noviembre del 2008, http://scientific. thomsonreuters.com/products/jcr/.).

Thomson Reuters. 2008d. Web of Science. (Consultado: 19 de abril del 2008, isiknowledge.com/.).

Thomson Scientific. 2008. Journal Scitation Report. (Consultado: 8 noviembre del 2008, http://scientific.thomsonreuters.com/products/jcr/.). 
